\title{
Identification of Secreted Exoproteome Fingerprints of Highly-Virulent and Non-Virulent Staphylococcus aureus Strains
}

\author{
Emilia Bonar ${ }^{1}$, Iwona Wojcik ${ }^{1}$, Urszula Jankowska ${ }^{2}$, Sylwia Kedracka-Krok ${ }^{2,3}$, \\ Michal Bukowski ${ }^{1}$, Klaudia Polakowska ${ }^{4}$, Marcin W. Lis ${ }^{5}$, Maja Kosecka-Strojek ${ }^{4}$, \\ Artur J. Sabat ${ }^{6}$, Grzegorz Dubin ${ }^{2,4}$, Alexander W. Friedrich ${ }^{6}$, Jacek Miedzobrodzki ${ }^{4}$, \\ Adam Dubin ${ }^{1}$ and Benedykt Wladyka ${ }^{1 *}$ \\ ${ }^{1}$ Department of Analytical Biochemistry, Faculty of Biochemistry, Biophysics and Biotechnology, Jagiellonian University, \\ Krakow, Poland, ${ }^{2}$ Malopolska Centre of Biotechnology, Jagiellonian University, Krakow, Poland, ${ }^{3}$ Department of Physical \\ Biochemistry, Faculty of Biochemistry, Biophysics and Biotechnology, Jagiellonian University, Krakow, Poland, ${ }^{4}$ Department \\ of Microbiology, Faculty of Biochemistry, Biophysics and Biotechnology, Jagiellonian University, Krakow, Poland, \\ ${ }^{5}$ Department of Veterinary and Animal Reproduction and Welfare, Faculty of Animal Welfare, University of Agriculture in \\ Krakow, Krakow, Poland, ${ }^{6}$ Department of Medical Microbiology, University of Groningen, University Medical Center \\ Groningen, Groningen, Netherlands
}

\section{OPEN ACCESS}

Edited by:

Martin John McGavin, University of Western Ontario, Canada

Reviewed by: Alexander Horswill, University of lowa, USA

Gerard Lina,

Centre International de Recherche en Infectiologie, France

*Correspondence:

Benedykt Wladyka benedykt.wladyka@uj.edu.pl

Received: 17 December 2015 Accepted: 19 April 2016 Published: 06 May 2016

Citation:

Bonar E, Wojcik I, Jankowska U, Kedracka-Krok S, Bukowski M,

Polakowska K, Lis MW, Kosecka-Strojek M, Sabat AJ, Dubin G, Friedrich AW,

Miedzobrodzki J, Dubin A and Wladyka B (2016) Identification of Secreted Exoproteome Fingerprints of

Highly-Virulent and Non-Virulent Staphylococcus aureus Strains.

Front. Cell. Infect. Microbiol. 6:51. doi: 10.3389/fcimb.2016.00051
Staphylococcus aureus is a commensal inhabitant of skin and mucous membranes in nose vestibule but also an important opportunistic pathogen of humans and livestock. The extracellular proteome as a whole constitutes its major virulence determinant; however, the involvement of particular proteins is still relatively poorly understood. In this study, we compared the extracellular proteomes of poultry-derived $S$. aureus strains exhibiting a virulent (VIR) and non-virulent (NVIR) phenotype in a chicken embryo experimental infection model with the aim to identify proteomic signatures associated with the particular phenotypes. Despite significant heterogeneity within the analyzed proteomes, we identified alpha-haemolysin and bifunctional autolysin as indicators of virulence, whereas glutamylendopeptidase production was characteristic for non-virulent strains. Staphopain C (StpC) was identified in both the VIR and NVIR proteomes and the latter fact contradicted previous findings suggesting its involvement in virulence. By supplementing NVIR, StpC-negative strains with StpC, and comparing the virulence of parental and supplemented strains, we demonstrated that staphopain $\mathrm{C}$ alone does not affect staphylococcal virulence in a chicken embryo model.

\section{Keywords: chicken embryo model, pathogen, protein, proteomics, Staphylococcus, virulence}

\section{INTRODUCTION}

Staphylococci constitute an important component of physiological biocenosis of skin and nose vestibule mucous membranes in man and anterior nares in animals (Devriese, 1990; Chen and Tsao, 2013). However, staphylococci are also dangerous opportunistic pathogens responsible for multiple infections (Bohach et al., 1990; Lowy, 1998). Among many species of staphylococci, Staphylococcus aureus receives major attention as an etiologic factor of human and livestock disease with rapidly increasing antibiotic resistance (Chambers and Deleo, 2009). 
Recent data demonstrates that although $S$. aureus infects many different host species, particular strains are adapted to certain hosts only (McCarthy et al., 2014). Species-specific adaptation is associated with acquisition and/or loss of mobile genetic elements (MGEs) (Malachowa and DeLeo, 2010; Lindsay, 2014) but also with reorganization of the entire genomes (Sung et al., 2008; Smyth et al., 2009; Hata et al., 2010). In line with the above, our previous study demonstrated that clear differences in virulence between avian strains as evaluated in a chicken embryo model are not reflected in a nematode model where generally low virulence and only minor differences between the evaluated strains were observed. Moreover, the significant differences in virulence in the embryo model correlated with the strain genotype, which was not the case in the nematode model (Polakowska et al., 2012). This suggests species-specific adaptations in the repertoire of virulence determinants. Several studies provide examples of host specific adaptations, including the arginine catabolic mobile element (ACME), which enhances survival in the human host (Diep et al., 2006, 2008; Barbier et al., 2010) or additional ruminant and horse-specific alleles of the von Willebrand factor-binding protein (Viana et al., 2010) modulating virulence specifically in these species. It has also been shown that human-to-poultry host specificity jump was associated with pseudogenization of the spa gene and acquisition of avian specific MGEs, among others a 17-kb pAvX plasmid encoding a thiol protease StpC (Takeuchi et al., 1999, 2002; Lowder et al., 2009). However, since host adaptation is clearly reflected in the organization of the entire genomes (Sung et al., 2008; Smyth et al., 2009; Hata et al., 2010), many adaptive processes are yet to be discovered.

Most of the identified host specific adaptations and virulence determinants are associated with the plasticity of the extracellular proteome, the primary site of host-pathogen interaction. However, despite many years of research effort and a few successful examples, we still poorly understand the role of particular exoproteins. The first major reason is the high genetic variability among staphylococci (Moore and Lindsay, 2001), suggesting that in most cases certain combinations of multiple factors rather than the expression of a particular one are involved in virulence, while there were no tools to analyze such complex systems until relatively recently (Becker and Bubeck Wardenburg, 2015). The second key reason is that the research is mostly focused on the interactions with the human host, while perforce animal models are used to verify the role of particular factors resulting in contradictory observations (Melehani et al., 2015; Shukla et al., 2015; Spaan et al., 2015).

In this study, we therefore chose to overcome these major issues by applying a holistic proteomic approach to identify virulence signatures within extracellular proteomes of avian strains carefully characterized in terms of their pathogenic potential in a chicken embryo model.

\section{MATERIALS AND METHODS}

\section{Bacterial Strains and Growth Conditions}

The exoproteome of poultry-derived $S$. aureus strains exhibiting either high $(\mathrm{CH} 3, \mathrm{CH} 5, \mathrm{CH} 9, \mathrm{CH} 23$, and $\mathrm{PA} 2)$ or low (ph1, ch22, pa3, ch24, ph2) virulence in a chicken embryo experimental infection model was analyzed. The detailed genetic characteristics, including sequence types (ST), agr status, phylogenetic relationships, and virulence data concerning the strains used in this study were described previously (Polakowska et al., 2012). S. aureus strains RN4220 (Kreiswirth et al., 1983), Newman (Duthie and Lorenz, 1952), ph1, and ch24 carrying a pALCP2 control plasmid (Bukowski et al., 2013) or a pALCP2/stpC plasmid driving the expression of a cysteine protease, staphopain C (StpC), were obtained by electroporation. Characteristics of the strains used is shown in Table 1.

For exoproteome analysis, the bacteria were cultured in tryptic soy broth (TSB) for $16 \mathrm{~h}$ at $37^{\circ} \mathrm{C}$ with vigorous shaking. Supernatants were collected by centrifugation ( $30 \mathrm{~min}, 15,000 \mathrm{~g}$, $4^{\circ} \mathrm{C}$ ) and decanting, repeated twice to ensure disposal of bacterial cells. For assessment of virulence, the bacteria were prepared as described previously (Polakowska et al., 2012), save that the media were supplemented with chloramphenicol $(10 \mu \mathrm{g} / \mathrm{ml})$ to ensure pALCP2 plasmid maintenance.

The cultures were conducted in three biological replicates for each strain in the same experimental conditions.

\section{Proteomic Analysis}

Cleared culture supernatants were incubated with an equal volume of $20 \%(\mathrm{w} / \mathrm{v})$ trichloroacetic acid (TCA) in acetone at $-20^{\circ} \mathrm{C}$ for $24 \mathrm{~h}$. Precipitated proteins were recovered by centrifugation $\left(25 \mathrm{~min}, 18,000 \mathrm{~g}, 4^{\circ} \mathrm{C}\right)$ and the resulting pellet was washed tree times with ice-cold acetone and air dried. The samples were dissolved in lysis buffer $(30 \mathrm{mM}$ Tris, $7 \mathrm{M}$ urea, $2 \mathrm{M}$ thiourea, 4\% CHAPS) and the total protein concentration was quantified using Quick Start Bradford reagent (Biorad). The protein samples from the virulent and nonvirulent strains as well as the internal standard, prepared by combining the protein samples from all tested replicates of virulent and non-virulent strains, were labeled with spectrally resolvable fluorescent dyes (Cy3, Cy5, and Cy2, respectively; GE Healthcare). The exoproteomes were compared within 12 pairs of virulent and non-virulent strains as summarized in Figure 1. The labeled samples of exo-proteins from the compared virulent strain, non-virulent strain, and the internal standard were combined and subjected to two-dimensional difference gel electrophoresis (2D DIGE) (Alban et al., 2003; Timms and Cramer, 2008; Minden et al., 2009). For each pair of the analyzed strains, in total $45 \mu \mathrm{g}$ of fluorescently labeled proteins were loaded on $7 \mathrm{~cm}$ immobilized $\mathrm{pH}$ gradient strips (IPG) by in-gel rehydratation. Isoelectrofocusing (IEF) was carried out using Protean IEF Cell (Bio-Rad), while proteins were separated in the second dimension in $12 \%$ acrylamide gel according to the Laemmli method (Laemmli, 1970). The gels were scanned using Typhoon Trio + (GE) and gel images were analyzed with Image Quant v.7.0 and DeCyder 2D software v.7.2 (GE). Subsequently, the gels were silver stained (Shevchenko et al., 1996) and the differentiating spots were excised and identified using mass spectrometry. 
TABLE 1 | Genetic and phenotypic characteristics of $S$. aureus strains used in the study.

\begin{tabular}{|c|c|c|c|c|c|c|c|}
\hline Strain & MLST type & spa type & agr group & $\operatorname{agr~status}^{a}$ & Virulence in chicken embyo model ${ }^{\mathbf{b}}$ & Proteolysis & Haemolysis \\
\hline $\mathrm{CH} 3$ & 5 & t002 & $\|$ & Pos & yes & + & ++ \\
\hline $\mathrm{CH} 5$ & 5 & t002 & $\|$ & Pos & yes & + & ++ \\
\hline $\mathrm{CH} 9$ & 5 & t002 & $\|$ & Pos & yes & +++ & - \\
\hline ch22 & 5 & t002 & $\|$ & Neg & no & +++ & + \\
\hline $\mathrm{CH} 23$ & 5 & t3478 & $\|$ & Pos & yes & +++ & ++ \\
\hline ch24 & 1 & t002 & III & Pos & no & + & + \\
\hline ch24/pALCP2 & 1 & t002 & III & ND & C & + & ND \\
\hline ch24/pALCP2/stpC & 1 & t002 & III & ND & c & +++ & ND \\
\hline Newman/pALCP2 & 2125 & t008 & I & ND & C & - & ND \\
\hline Newman/pALCP2/stpC & 2125 & t008 & I & ND & C & +++ & ND \\
\hline PA2 & 1346 & t002 & $\|$ & Pos & yes & +++ & + \\
\hline pa3 & 692 & t8646 & 1 & Pos & no & - & - \\
\hline ph1 & 1347 & t8646 & 1 & Pos & no & - & - \\
\hline ph1/pALCP2 & 1347 & t8646 & 1 & ND & c & - & ND \\
\hline ph1/pALCP2/stpC & 1347 & t8646 & 1 & ND & C & ++ & ND \\
\hline ph2 & 692 & t8646 & 1 & Pos & no & +++ & - \\
\hline RN4220/pALCP2 & 8 & t211 & 1 & $N D^{d}$ & c & - & ND \\
\hline RN4220/pALCP2/stpC & 8 & t211 & 1 & $\mathrm{ND}^{\mathrm{d}}$ & C & +++ & ND \\
\hline
\end{tabular}

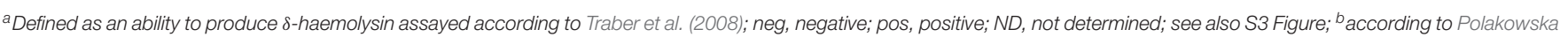

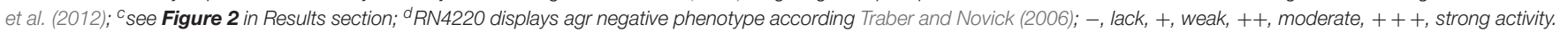

\section{Mass Spectrometry Identification of Proteins}

The excised gel fragments were destained by several subsequent washes in 25 and $50 \%(\mathrm{v} / \mathrm{v})$ acetonitrile (ACN) in $25 \mathrm{mM}$ ammonium bicarbonate buffer $\left(\mathrm{NH}_{4} \mathrm{HCO}_{3}\right), \mathrm{pH} 8.0$ at $37^{\circ} \mathrm{C}$. Next, the gel fragments were dehydrated in $\mathrm{ACN}$, dried using a vacuum concentrator, and rehydrated using $15 \mu \mathrm{L}$ of trypsin (Biocentrum) solution $\left(10 \mathrm{ng} / \mu \mathrm{L}\right.$ in $25 \mathrm{mM} \mathrm{NH}_{4} \mathrm{HCO}_{3}$, $\mathrm{pH}$ 8.0) for $15 \mathrm{~min}$. Additional $20 \mu \mathrm{L}$ of $25 \mathrm{mM} \mathrm{NH}_{4} \mathrm{HCO}_{3}$ buffer were added and digestion was carried out overnight at $37^{\circ} \mathrm{C}$. Peptides were extracted by sonication and dehydrated in ACN. The extracts were evaporated to dryness using a vacuum concentrator and suspended in $2 \%(\mathrm{v} / \mathrm{v})$ ACN in water containing $0.05 \%(\mathrm{v} / \mathrm{v})$ trifluoroacetic acid (TFA). The resulting peptides were separated on a $15 \mathrm{~cm} \times 75$ $\mu \mathrm{m}$ RP column $(2 \mu \mathrm{m}$, Acclaim PepMap $75 \mu \mathrm{m} 100 \AA$ Nano Series TM Column) using a $2-40 \%$ gradient of ACN in $0.05 \%$ formic acid during $30 \mathrm{~min}$ on UltiMate 3000RS LCnanoSystem (Dionex). The system additionally contained a $\mathrm{C}_{18}$ precolumn $(3 \mu \mathrm{m}, 2 \mathrm{~cm} \times 75 \mu \mathrm{m}$ Acclaim PepMap Nano trap Column). The peptides were analyzed on a coupled MicrOTOF-QII mass spectrometer (Bruker) equipped with an Appollo Source ESI nano-sprayer with a low-flow nebulizer. The MS was operated in the standard data-dependent acquisition MS/MS mode with fragmentation of most intensive precursor ions.

\section{Analysis of Mass Spectrometry Data}

MS spectra were recalibrated using fragment ions of trypsinderived peptides. Raw data was pre-processed with Data
Analysis 4.0 software (Bruker, Germany) into the Mascot Generic format. The SwissProt_201202 non-redundant protein database taxonomically restricted to Firmicutes (gram-positive bacteria; 68048 sequences) was queried with the obtained peak lists using an in-house Mascot server (v.2.3.0, Matrix Science, London, UK). The following search parameters were applied: permitted number of missed cleavages-one, fixed modification-carbamidomethylation (C), variable modification-oxidation, protein mass-unrestricted, peptide mass tolerance $- \pm 20 \mathrm{ppm}$, fragment mass tolerance - \pm 0.05 Da. Only identifications with a score value over 100 were considered relevant for further analysis. If more than one protein was identified in one spot, only those with the scores of over $50 \%$ of the highest scoring protein were considered in further analysis.

\section{Virulence Assessment in a Chicken Embryo Model}

The experiments were performed in compliance with the animal protection laws of Poland. Experiments using chicken embryos were terminated on development day 17 at the latest, 4 days prior to hatching. The virulence of each tested strain was assessed as described previously (Polakowska et al., 2012). In brief, a titrated suspension of bacteria of a tested strain was inoculated to embryos at the 10th day of their development and then the viability of embryos was monitored for the next 7 days by candling. All experiments were repeated in three independent runs. In each run, the effect of each strain was evaluated at two dilutions: $10^{6}$ and $10^{4} \mathrm{CFU} /$ egg. Each run involved 20 eggs for each bacterial dilution and the control. 
A
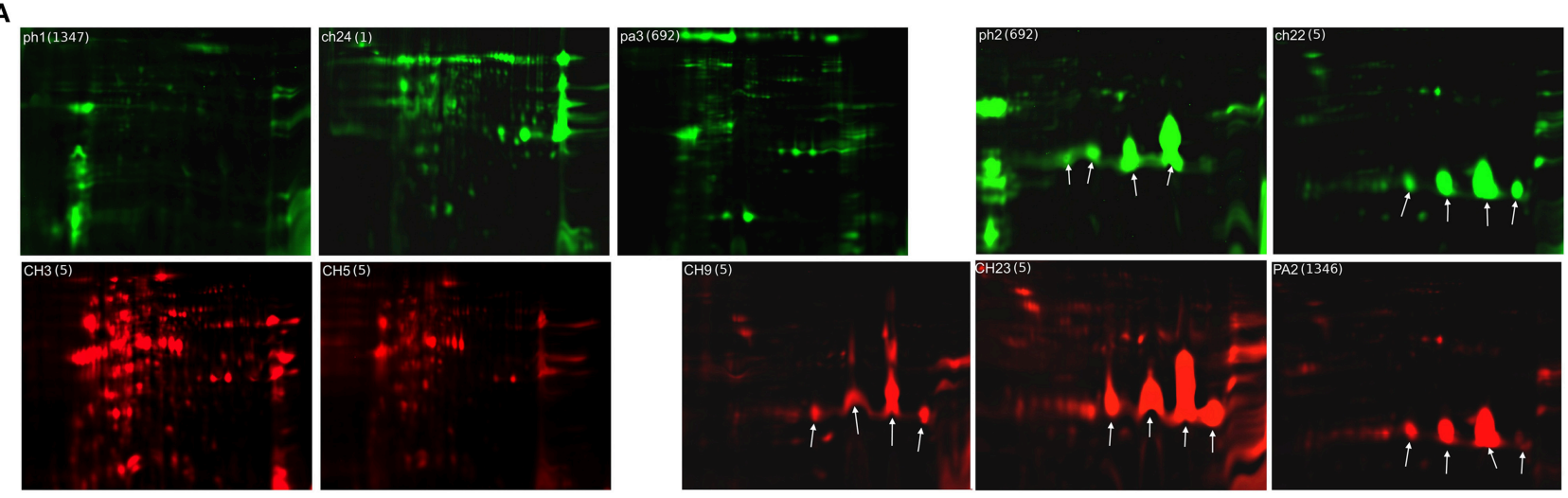

B

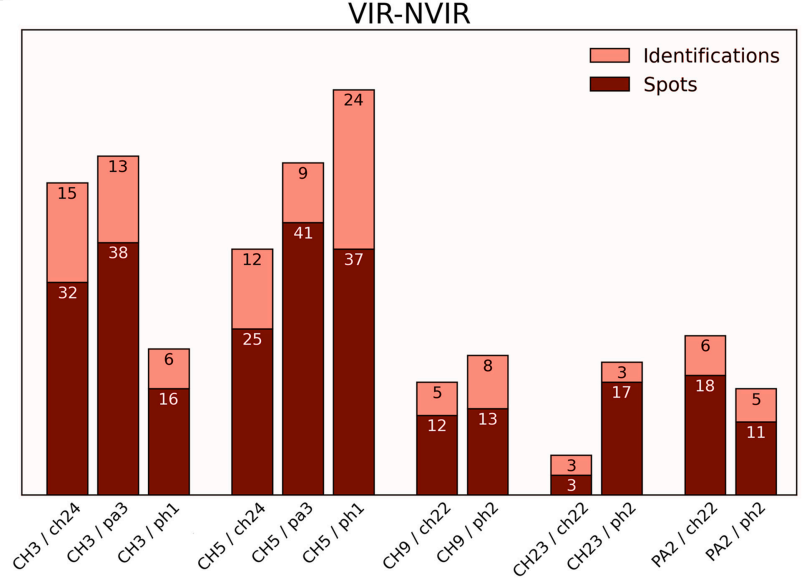

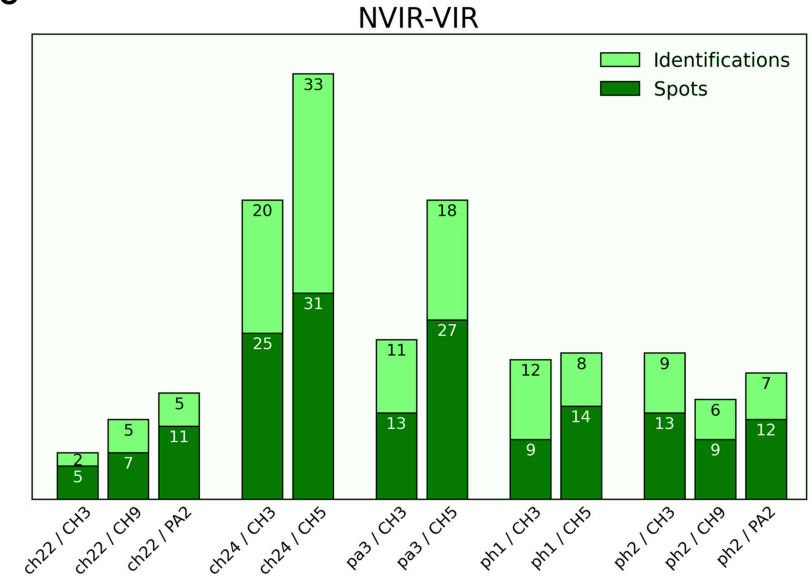

FIGURE 1 | Staphylococcal exoproteome profiles analyzed by 2D gel electrophoresis and mass spectrometry (MS). (A) The exoproteomes of poultry-derived S. aureus strains exhibit two major types of profiles: (right) dominated by staphopain C (StpC; arrows) expression or (left) lacking the expression of staphopain $\mathrm{C}$. The profile type does not correlate with virulence in the chicken embryo model-virulent strains $\mathrm{CH} 3, \mathrm{CH}$, $\mathrm{CH}$, $\mathrm{CH} 23$, $\mathrm{PA} 2$ (red); non-virulent strains ph1, ch22, pa3, ch24, ph2 (green). Molecular weight range is 6-116 kDa, pl range is 3-10 for gels without staphopain C (CH3, CH5, ph1, ch24, and pa3) and from 4 to 7 in case of gels with $\mathrm{StpC}(\mathrm{CH} 9, \mathrm{CH} 23, \mathrm{PA} 2$, ph2, and ch22). Sequence type (ST) is provided next to the strain tag in a bracket. (B,C) respectively, summary of 2D DIGE and MS identification analyses of the staphylococcal exoproteomes of the virulent and non-virulent strains (dark color bar-number of differentiating spots cut out from the gel, light color bar-number of proteins identified by MS analysis; the differences in the above referenced numbers arise mainly from the fact that in many cases multiple spots originated from a single protein).

\section{RESULTS}

\section{The Extracellular Proteomes of Virulent and Non-Virulent S. aureus Strains Are Highly Heterogeneous}

We have recently demonstrated a clear correlation between the genotype and the virulence level as evaluated in the chicken embryo model within a collection of poultry-derived $S$. aureus strains (Polakowska et al., 2012). In this study, we intended to elucidate how the observed genetic differences determining the virulence level translated into differences within respective extracellular proteomes. To this end, of the strains characterized in our previous study, five virulent (VIR) ones were chosen $(\mathrm{CH} 3$, $\mathrm{CH} 5, \mathrm{CH} 9, \mathrm{CH} 23$, and PA2) and their extracellular proteomes were analyzed and compared to those of the five previously characterized non-virulent (NVIR) strains (ph1, ch22, pa3, ch24, and $\mathrm{ph} 2$ ). The extracellular proteomes of all tested strains were analyzed by two-dimensional polyacrylamide gel electrophoresis (2D-PAGE) demonstrating significant variability both between the VIR and NVIR groups and within each group (results not shown). To identify the components of the extracellular proteome possibly involved in virulence, we paired the VIR strains with those belonging to the NVIR group according to the overall similarities within the electrophoretic patterns and compared their secreted proteomes using difference gel electrophoresis (DIGE). Twelve pairs were analyzed in total allowing comparison of each virulent strain with either two or three non-virulent counterparts (Figure 1, S1, S2 Tables). Analysis of DIGE gel images confirmed high variation in the number and location of differentiating protein spots among the virulent strains (from 20 spots identified in $\mathrm{CH} 23$, paired with ch22 and ph2, up to 103 spots for CH5, paired with pa3, ph1, and ch24; Figure 1B). Comparable variation was observed for the non-virulent strains (from 23 spots in ph1 to 56 in ch24, 
both paired with $\mathrm{CH} 3$ and $\mathrm{CH} 5$; Figure 1C). As in case of the high variability in the electrophoretic patterns of the analyzed exoproteomes, the number of protein spots differentiating the VIR and NVIR exoproteomes in the analysis of particular pairs was also highly variable, ranging from eight spots differentiating the proteomes of $\mathrm{CH} 23 / \mathrm{ch} 22$ up to 64 spots in the $\mathrm{CH} 5 / \mathrm{pa} 3$ pair, even despite the fact that, prior to comparative analysis, the strains were paired based on the overall similarity of respective electrophoretic patterns. Interestingly, a majority of the identified differences in the exoproteomes of the VIR and NVIR strains were of a qualitative nature. Moreover, the total number of spots identified as differentiating in all the VIR strains (263) was substantially higher than of those in the NVIR strains (176).

\section{Staphopain C Overexpression Does Not Affect the Virulence Level}

One striking finding of the electrophoretic analysis reported above was that the exoproteomes of certain strains are characterized by four major spots almost exclusively dominating the electrophoretic pattern, which indicates strong overexpression of those proteins. LC-MS/MS analysis demonstrated that all those spots originate from a single protein-a cysteine protease, staphopain C (StpC) (Figure 1A). The protease is encoded in the $\mathrm{pAvX}$ plasmid, which is a part of the poultry-specific pool of accessory genetic material, and has been suggested to play a role in poultry dermatitis (Kuramasu et al., 1967; Takeuchi et al., 2002; Lowder et al., 2009). Interestingly, however, we observed StpC overexpression in strains belonging to both VIR ( $\mathrm{CH} 9, \mathrm{CH} 23$, and PA2) and NVIR (ph2 and ch22) groups. To analyze the discrepancy between the above data and our own observations, we verified the influence of StpC on the virulence of different strains in our chicken embryo model. To this end, the non-virulent strains, including RN4220 and Newman of human origin and two poultry associated strains (ch24 and ph1; neither of which contained pAvX plasmid), were supplemented with a plasmid carrying a StpC encoding cassette under its native promoter or an empty plasmid. Before supplementation all non-virulent strains exhibited low proteolytic activity as determined using skim milk agar plate assay. In the same assay, the supplemented strains exhibited high level of proteolytic activity, comparable to wild-type StpC producers. The virulence of recombinant strains was compared in the chicken embryo model. No significant difference in the survival rate was observed in any of the tested pairs between a particular strain supplemented with the StpC expressing plasmid or an empty one (Figure 2). These results clearly indicate that StpC expression alone does not determine the virulence level in the chicken embryo model.

\section{Alpha-Haemolysin (HLA) and Bifunctional Autolysin (ATL) Constitute the Fingerprint of a Virulent Proteome}

Our further proteomic analysis aimed at finding correlations, if any, between the expression of particular exoproteins and the virulence in the chicken embryo model. Twochannel analysis of DIGE gels identified 263 protein spots characteristic for VIR strains only, within the 12 pairs of compared VIR and NVIR exoproteomes. LC-MS/MS analysis demonstrated that the spots represent 46 different staphylococcal proteins, as multiple spots originated from the same protein, suggesting posttranslational modifications (Table 2 and S3 Table). Of those proteins, only alpha-haemolysin (HLA) and bifunctional autolysin (ATL) were identified in all five analyzed VIR strains. This could indicate that these two proteins constitute a core of a virulent proteome; nevertheless, further analysis demonstrated that the interpretation is more complicated (see below). Formate-tetrahydrofolate ligase (FTHS) and phosphoenolpyruvate carboxykinase (PCKA) were identified in the exoproteomes of all but $1(\mathrm{CH} 23)$ virulent strains. Fifteen other proteins were identified each in two VIR strains and 27 further proteins were characteristic for single analyzed VIR exoproteomes.

One-channel analysis of the exoproteomes clearly demonstrated two major types of patterns in 2-DE. The first type is characterized by a large number of spots, none of which strongly predominates ( $\mathrm{CH} 3, \mathrm{CH} 5, \mathrm{ph} 1, \mathrm{ch} 24$, pa3). The second type shows a relatively small number of spots with several large spots dominating the image $(\mathrm{CH} 9, \mathrm{CH} 23, \mathrm{PA} 2$, ph2, ch22) (Figure 1A). When the VIR and NVIR proteomes are compared within the first type, HLA, enterotoxin type D (ETXD), lipase 1 (LIP1), lipase 2 (LIP2), and N-acetylmuramoylL-alanine amidase domain-containing protein (Y2979) are found in all VIR strains. Interestingly, these proteins were identified with extraordinary frequency $(16 ; 13 ; 17 ; 18$; and 7 identifications for $\mathrm{CH} 3$, and $18 ; 6 ; 30 ; 28$; and 13 for $\mathrm{CH} 5$, respectively), as the number of identifications of those five proteins constitutes 74.7 and $69.3 \%$ of all protein identifications in the exoproteomes of the $\mathrm{CH} 3$ and $\mathrm{CH} 5$ strains, respectively. When VIR and NVIR proteomes are compared within the second type, none of the additional proteins characteristic for all VIR strains are identified apart from ATL and HLA mentioned previously. However, for the $\mathrm{CH} 9$ and $\mathrm{PA} 2$ virulent strains, PCKA, putative surface protein SAV2496 (PLS), and surface protein G (SASG) were identified as upregulated regardless of the non-virulent strain (ch22 or ph2) used for comparison. These proteins also constituted a major part of the exoproteome, 67.7 and $83.3 \%$ of all protein identifications in $\mathrm{CH} 9$ and PA2, respectively.

\section{Glutamylendopeptidase Expression Is a Fingerprint of a Non-Virulent Exoproteome}

One hundred and seventy six protein spots were detected as positively differentiating in non-virulent exoproteomes within 12 performed comparisons with the virulent counterparts. These spots originated from 66 different proteins, as identified by MS analysis. Glutamylendopeptidase (SSPA, also referred to as V8 protease) expression differentiated all analyzed NVIR proteomes compared to those of virulent strains (Table 3 and S4 Table). Expression of alcohol dehydrogenase $(\mathrm{ADH})$, alkyl hydroperoxide reductase subunit C (AHPC), and 
TABLE 2 | A list of proteins identified when analyzing the spots differentiating (up regulated) in VIR strains exoproteomes compiled according to the number of strains with identifications within the group of five VIR strains.

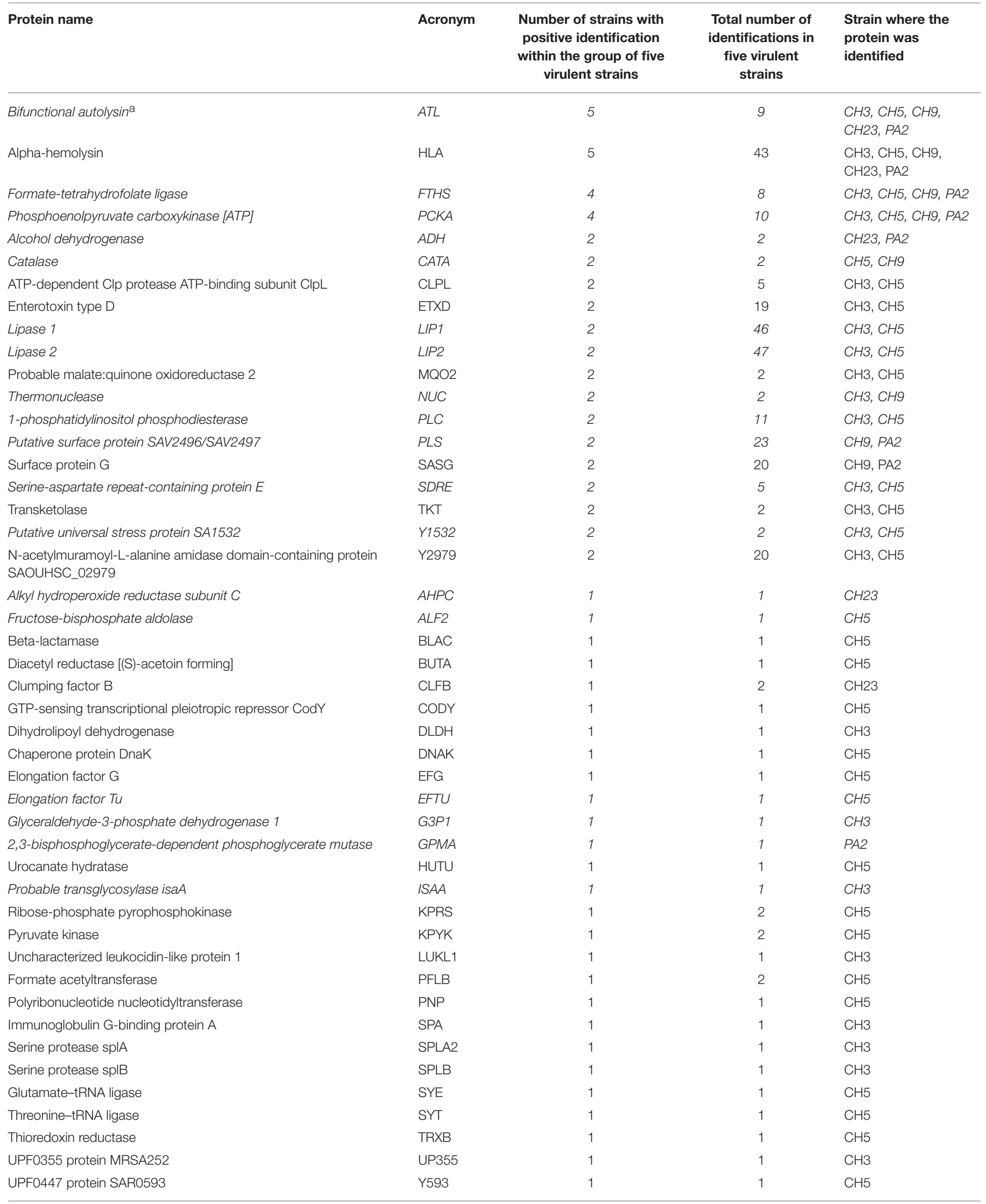

a Proteins identified as differentiating in both VIR and NVIR strain proteomes are italicized (see also subchapter "Analysis of non-overlapping spots with identical identification"). 
TABLE 3 | A list of proteins identified when analyzing the spots differentiating (up regulated) in NVIR strains exoproteomes compiled according to the number of strains with identifications within the group of five NVIR strains.

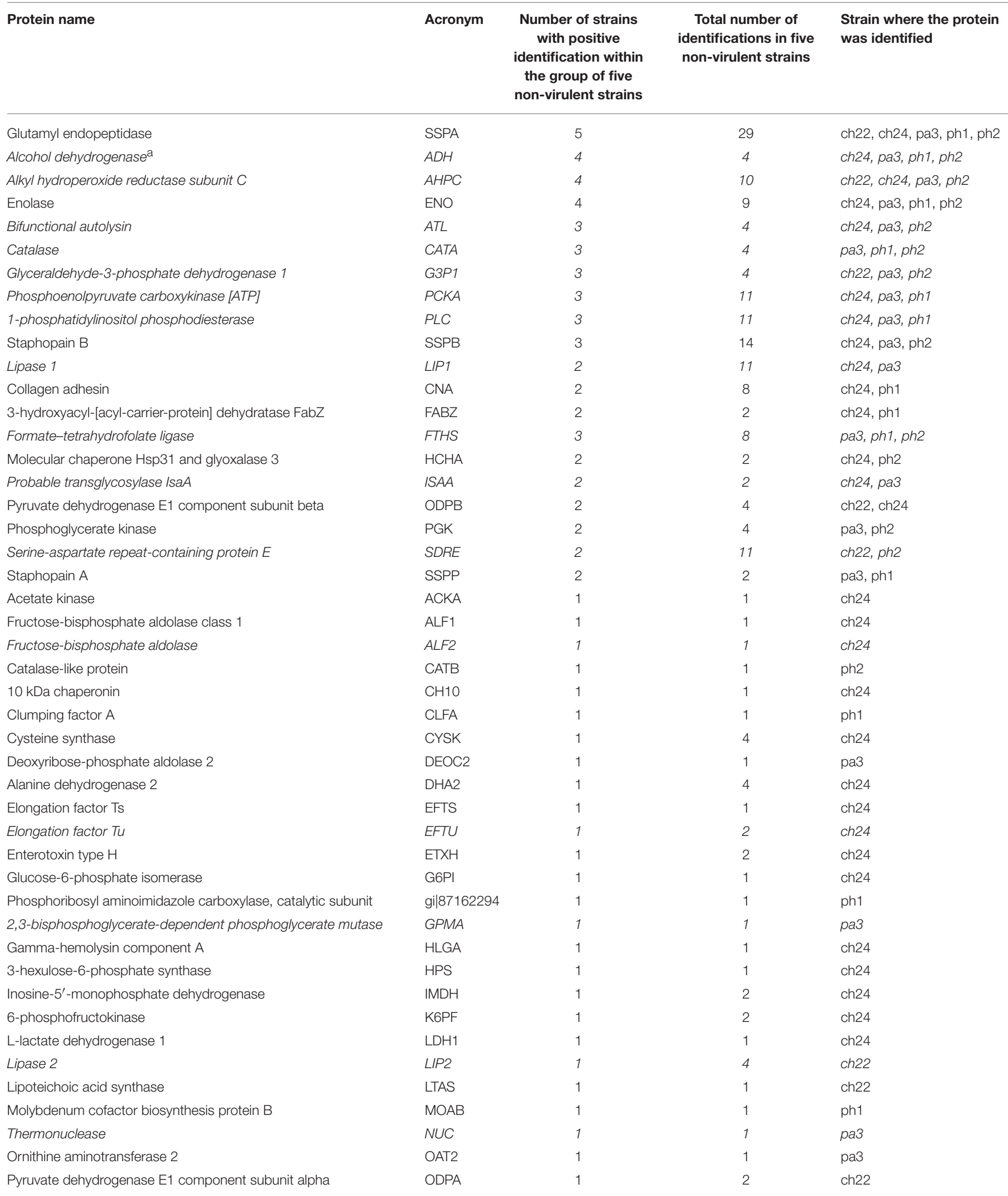




\begin{tabular}{|c|c|c|c|c|}
\hline Protein name & Acronym & $\begin{array}{l}\text { Number of strains } \\
\text { with positive } \\
\text { identification within } \\
\text { the group of five } \\
\text { non-virulent strains }\end{array}$ & $\begin{array}{c}\text { Total number of } \\
\text { identifications in five } \\
\text { non-virulent strains }\end{array}$ & $\begin{array}{l}\text { Strain where the protein } \\
\text { was identified }\end{array}$ \\
\hline Putative surface protein SAV2496/SAV2497 & PLS & 1 & 1 & ch24 \\
\hline Putative peptidyl-prolyl cis-trans isomerase & PPI1 & 1 & 1 & pa3 \\
\hline Phosphate acetyltransferase & PTAS & 1 & 1 & pa3 \\
\hline Phosphocarrier protein $\mathrm{HPr}$ & PTHP & 1 & 2 & ph1 \\
\hline $50 S$ ribosomal protein L14 & RL14 & 1 & 1 & pa3 \\
\hline 50S ribosomal protein L5 & RL5 & 1 & 1 & pa3 \\
\hline 30S ribosomal protein S8 & RS8 & 1 & 2 & pa3 \\
\hline Superoxide dismutase [Mn/Fe] 1 & SODM1 & 1 & 2 & ch24 \\
\hline Prophage-derived single-stranded DNA-binding protein & SSBP & 1 & 1 & ph1 \\
\hline Phenylalanine-tRNA ligase alpha subunit & SYFA & 1 & 2 & ch24 \\
\hline Signal transduction protein TRAP & TRAP & 1 & 1 & ph1 \\
\hline Uracil phosphoribosyltransferase & UPP & 1 & 1 & ch24 \\
\hline Uncharacterized N-acetyltransferase SA1019 & Y1019 & 1 & 1 & ch24 \\
\hline Putative universal stress protein SA1532 & Y1532 & 1 & 2 & pa3 \\
\hline UPF0173 metal-dependent hydrolase SAB1566c & Y1566 & 1 & 1 & ch24 \\
\hline Uncharacterized protein SA1692 & Y1692 & 1 & 1 & ch24 \\
\hline Uncharacterized oxidoreductase SAS2370 & Y2370 & 1 & 1 & ch24 \\
\hline Uncharacterized protein SA0829 & Y829 & 1 & 1 & ch24 \\
\hline UPF0477 protein SA0873 & Y873 & 1 & 1 & ch24 \\
\hline Uncharacterized protein SAOUHSC_00997 & Y997 & 1 & 3 & ch24 \\
\hline
\end{tabular}

aproteins identified as differentiating in both VIR and NVIR strain proteomes are italicized (see also subchapter "Analysis of non-overlapping spots with identical identification").

enolase (ENO) was detected in four out of the five nonvirulent exoproteomes. Expression of seven other proteins was detected in three NVIR strains. Another nine proteins were identified each in two NVIR strains. Finally, 46 proteins were identified each in a single NVIR strain, (Table 3 and S4 Table).

Analogically to the proteins characteristic for VIR exoproteomes described in the previous chapter, those characteristic for NVIR exoproteomes were also identified in multiple protein spots. This phenomenon was most pronounced for PCKA, PLC, LIP1, and cysteine protease staphopain B (SSPB) (each identified in at least ten different spots in the analyzed exoproteomes). Moreover, serine-aspartate repeat-containing protein E (SDRE) was identified within nine different spots in strain ch22 exoproteome compared with the $\mathrm{CH} 9, \mathrm{CH} 23$, and PA3 virulent strains.

Glutamylendopeptidase (SSPA) was previously suggested to play a role in virulence of S. aureus (Coulter et al., 1998; Kolar et al., 2013). We therefore found it surprising that this enzyme was identified only in the exoproteomes of NVIR but not VIR strains, especially that the enzyme was abundantly expressed (29 spots in all analyzed exoproteomes originated from SSPA). It was demonstrated previously that SSPA is produced as a zymogen activated by a metalloprotease aureolysin. We therefore hypothesized that possibly it is not SSPA itself, but a deficiency in its activation that is characteristic for NVIR strains, especially that only mature SSPA activates SSPB zymogen which in turn was also suggested of importance in staphylococcal virulence (Rice et al., 2001). By analyzing sequence coverage in LC-MS/MS identifications and approximate molecular weights in 2-DE, we were able to distinguish, with relatively high confidence, spots belonging to the zymogen and the mature protease (S1 Figure). Seven SSPA-originating spots contained tryptic peptides encompassing a part of a profragment or an intact Asn68-Val69 zymogen processing site (Nickerson et al., 2007), directly demonstrating that these spots originated from the SSPA zymogen. In the remaining 22 spots originating from SSPA, none of the tryptic peptides corresponded to the profragment. The approximate molecular weight of proteins contained within most of those spots indicates that they originate form mature SSPA, therefore SSPA activation is not affected in these strains. The molecular weight of certain spots corresponded to the zymogen and here the identification was ambiguous. Moreover, several spots with approximate molecular weight below that expected for mature SSPA were found, in which the peptide coverage suggested processing at the Cterminus. Overall, the exoproteomes of strains ch22, ch24, and ph2 contained both the zymogen and the mature form of SSPA. Exclusively in ph1, only the mature form was detected. Interestingly, pa3 contained only SSPA zymogen, which may suggest impaired activation in this strain (Figure 3 and S1 Figure). The impaired activation cascade in pa3 is further supported by the fact that SSPB is also found in its zymogen form in this strain, whereas in other strains mature SSPA 


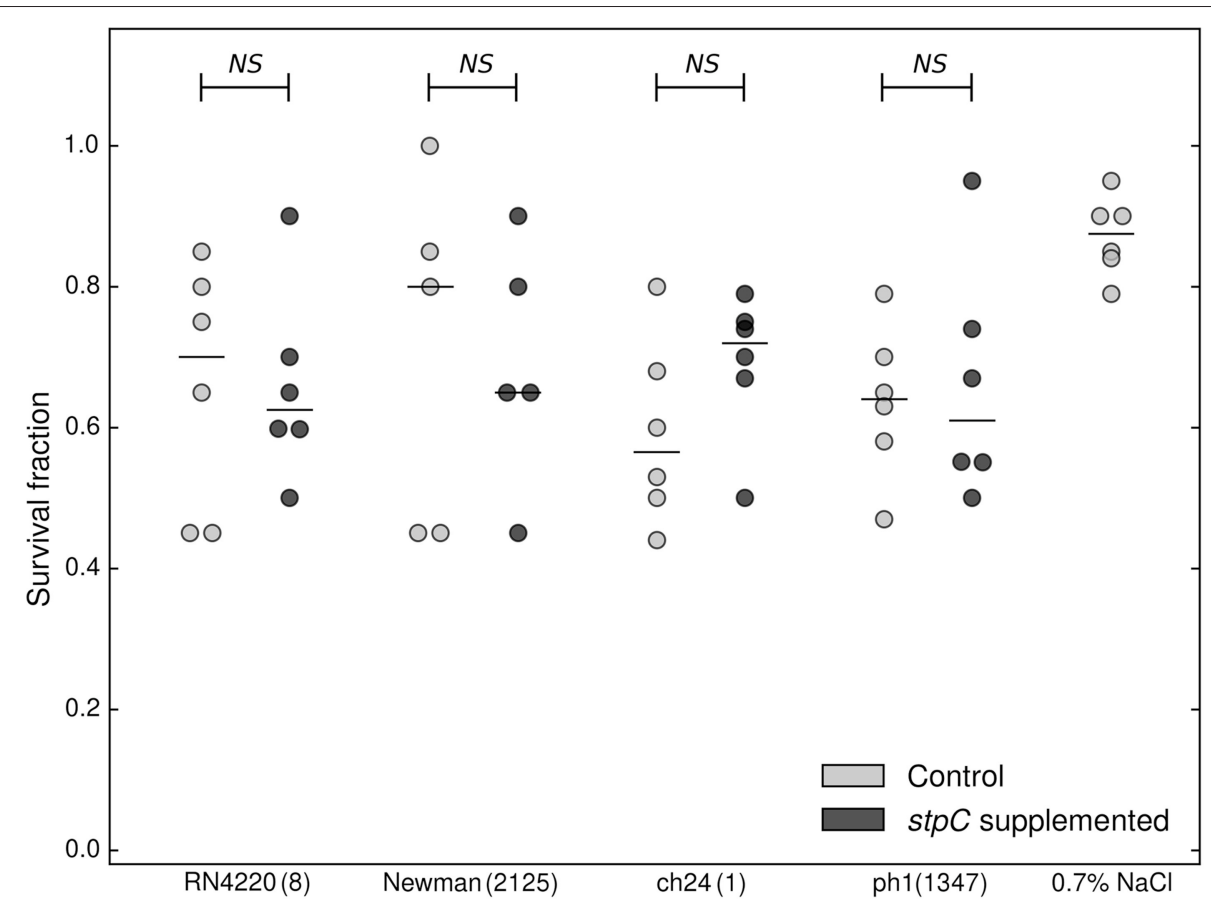

FIGURE 2 | Comparison of virulence of WT (stpC ${ }^{-}$, supplemented with a control plasmid) and staphopain C-supplemented staphylococcal strains in the chicken embryo model. Cumulative survival of chicken embryos 7 days following inoculation with staphylococcal strains supplemented with staphopain $\mathrm{C}$ expressing plasmid (dark gray circles), and control plasmid (light gray circles). The median is marked with a horizontal line. Each circle corresponds to an independent data point. No statistically significant differences were recorded (Mann-Whitney test). ST is provided next to the strain tag in a bracket.

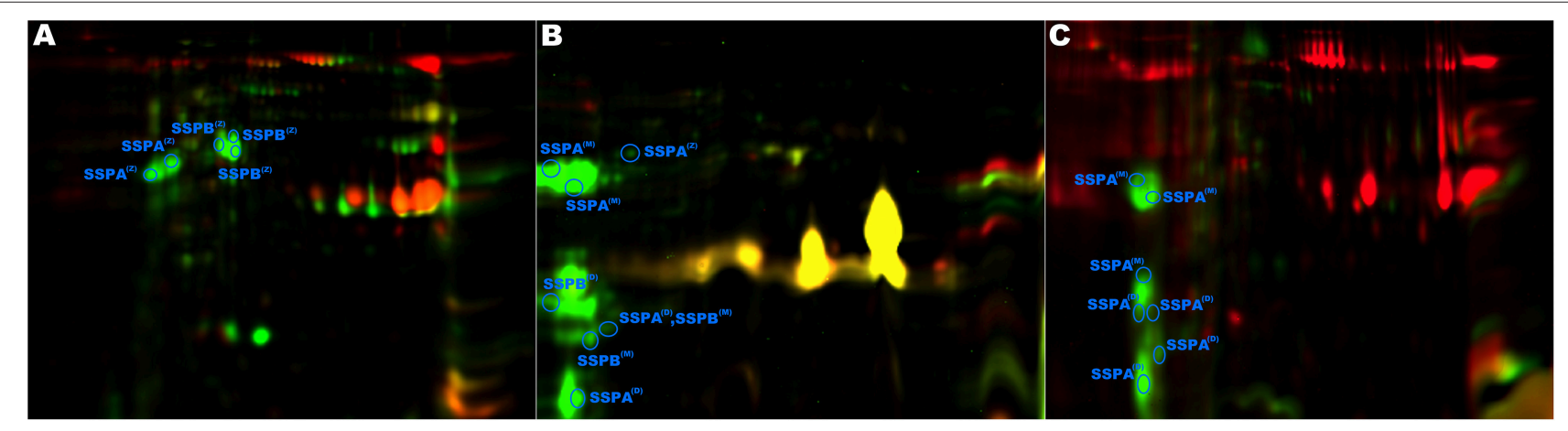

FIGURE 3 | Analysis of glutamylendopeptidase (SSPA) and staphopain B (SSPB) posttranslational processing. SSPA and SSPB were identified only in the non-virulent strains. (A) Strain pa3 contained only zymogen ( $Z$ in the superscript) forms of both proteases (as evidenced by approximate molecular weight and tryptic peptide coverage), indicating a disrupted activation pathway. Strains ph2 (B) and ph1 (C) contained the zymogen, the mature (M in the superscript) forms, and the degradation ( $\mathrm{D}$ in the superscript) products of the proteases.

correlates with mature SSPB, as expected according to the activation mechanism described previously (Nickerson et al., 2007).

\section{Analysis of Non-Overlapping Spots with Identical Identification}

2D-DIGE analysis is best suited for detecting relatively small differences between closely related proteomes. In this study, exoproteomes of distantly related strains were compared while 2D-DIGE was successfully used as a convenient means of eliminating proteins of housekeeping function or expressed regardless of the strain virulence (spots yellow and orange in color) from further analysis. To this end, only differentiating spots were subject to further analysis (red and green spots, reflecting proteins with higher abundance in VIR and NVIR proteomes, respectively). Interestingly, however, identical proteins were often detected in multiple spots, both within a single exoproteome (multiple spots with identical identification), but also in differential analysis of two proteomes (nonoverlapping spots with identical identification; italicized in 
Tables 2, 3). Such differences may reflect genetic variation or posttranslational modifications or both and may have an important impact on protein function. As such, the sources of the observed variability were analyzed in greater detail for LIP1, PLC, FTHS, PCKA, CATA, and ATL, since the highest heterogeneity was observed among those proteins (Figure $\mathbf{4}$ and S2 Figure).

Spots originating from LIP1 derived from different strains do not overlap because of the differences in $\mathrm{pI}$ (Figures $4 \mathrm{~A}, \mathrm{~B}, \mathrm{E}$ ). Sequence coverage in LC-MS/MS identification demonstrates that the analyzed spots originate from the LIP1 precursor. The LIP1 precursor derived from the NVIR strains (ch24 and pa3) is shifted toward higher $\mathrm{pI}$ relative to the LIP1 derived from the VIR strains ( $\mathrm{CH} 3$ and $\mathrm{CH} 5)$. To evaluate if genetic differences or posttranslational modifications are responsible, we compared the open reading frames encoding LIP1 obtained from shotgun sequencing of respective strains. The amino acid sequences of LIP1 derived from the VIR strains (calculated $\mathrm{pI}=6.21$ ) was identical to that derived from S. aureus Mu50 (ATCC 700699), chosen as the best reference sequence by the software for protein identification. In turn, the sequences derived from the NVIR strains differed by 9 and 7 amino acids, respectively, for strains ch24 and pa3 (calculated pI $=6.49$ and 6.31, respectively). Therefore, the partial non-overlap of spots originating from LIP1 is explained by differences in the amino acid sequence of the protein derived from different strains. The exoproteome of pa3 contains additional spots originating form LIP1, but located in a different part of the gel. Here, however, the approximate molecular weight and sequence coverage clearly demonstrate that these spots originate form intermediate and mature forms of LIP1.

Similarly to LIP1, allelic variants are responsible for shifting of the spots originating from PLC from the NVIR strains (ch24 and ph1) toward higher $\mathrm{pI}$, compared to those originating from the VIR strains (CH3 and CH5) (Figures 4A,C,D,F). The same is true for FTHS, although here the protein originating from the NVIR strain (pa3) is shifted toward lower pI compared to that derived from the VIR strain (CH5). In addition, FTHS is more abundantly expressed in pa3, compared to $\mathrm{CH} 5$ (Figure 4E). Similarly, in the case of PCKA (Figures 4C,E), the protein sequence and corresponding $\mathrm{pI}$ differs in the VIR and NVIR strains (calculated pI of 5,74 and 5,89, respectively). In parallel, proteolytic processing at the C-terminus of PCKA is observed in strain ph1 (Figure 4C), as evidenced by the slight shift to lower molecular weight and the peptide coverage obtained. Proteolytic processing at the C-terminus is also observed within CATA in the NVIR strain (pa3) but not in CH5 (Figure 4E), which in this case is solely responsible for the lack of co-localization of corresponding spots. Overall, this analysis concludes in an interesting observation that the exoproteomes of the VIR an NVIR strains not only differ in the protein content, but are also characterized by distinct genetic variants and differences in posttranslational processing. These phenomena may constitute yet another level of complexity necessary to uncover in order to better understand the role and interplay of virulence determinants; however, our limited sample does not allow establishing meaningful correlations.
When spots differentiating the VIR from NVIR strains were analyzed, bifunctional autolysin (ATL) was identified in all VIR exoproteomes. At the same time, analysis of spots differentiating the NVIR strains also resulted in identification of ATL. In this case, the difference in spot localization on 2D-gels was not related to genetic differences or simple processing. ATL is a large protein exhibiting two enzymatic activities. The N-terminal domain exhibits $\mathrm{N}$-acetylmuramoyl-L-alanine amidase activity, whereas the C-terminal part is an endo-beta- $\mathrm{N}$-acetylglucosaminidase (Gotz et al., 2014). The tryptic peptides identified in ATL containing spots originating from the VIR strains aligned almost exclusively (8/9) with the N-terminal part of ATL (amidase), while those from NVIR strains mostly (3/4) to the C-terminal part (glucosaminidase). This suggests that the amidase activity of ATL may be important in virulence; however, the mechanism determining differential stability of the two domains of a single protein and the role of the ATL amidase domain in pathogenesis, if any, remain unknown.

\section{DISCUSSION}

We demonstrated previously that, among poultry-derived $S$. aureus strains, those exhibiting high virulence in a chicken embryo model are genetically distinct from non-virulent strains (Polakowska et al., 2012). In this study, we applied proteomics to elucidate how genetic differences are reflected in the extracellular proteome and to identify proteomic signatures of virulent and non-virulent strains. Especially the latter were notoriously neglected in previous analyses, while the concept of "virulence attenuators" gains importance in understanding of commensal coexistence of opportunistic pathogens (Merhej et al., 2013).

The exoproteomes of five VIR and five NVIR strains were analyzed by $2 \mathrm{D}$-electrophoresis demonstrating two major exoprotein patterns which, however, did not follow the VIR/NVIR division. The exoproteomes were either dominated by the presence of StpC and contained relatively few other spots ( $\mathrm{CH} 9, \mathrm{CH} 23, \mathrm{PA} 2$, ph2, and ch22) or contained a large number of spots without any dominant ones (remaining strains). Interestingly, StpC was previously postulated as an avian hostspecific virulence factor (Lowder et al., 2009), which contradicts our findings. To further investigate this issue, we tested the effect of StpC supplementation on the virulence of a non-virulent, StpC-negative strains. We found that StpC supplementation was not sufficient to increase the virulence of the non-virulent strains, demonstrating that StpC alone does not significantly affect the virulence potential. However, these findings do not exclude the role of StpC in poultry-host preference.

We compared matched pairs of VIR and NVIR exoproteomes within the two groups defined above. Collectively, in these analyses, the number of spots characteristic for the VIR strains was higher than the number of those specific for NVIR strains. Interestingly, however, the spots characteristic for the VIR strains originated from a smaller number of individual proteins than those specific for the NVIR strains, which indicates significant posttranslational modification of proteins within the VIR strains. The identification of multiple spots within the VIR exoproteomes 

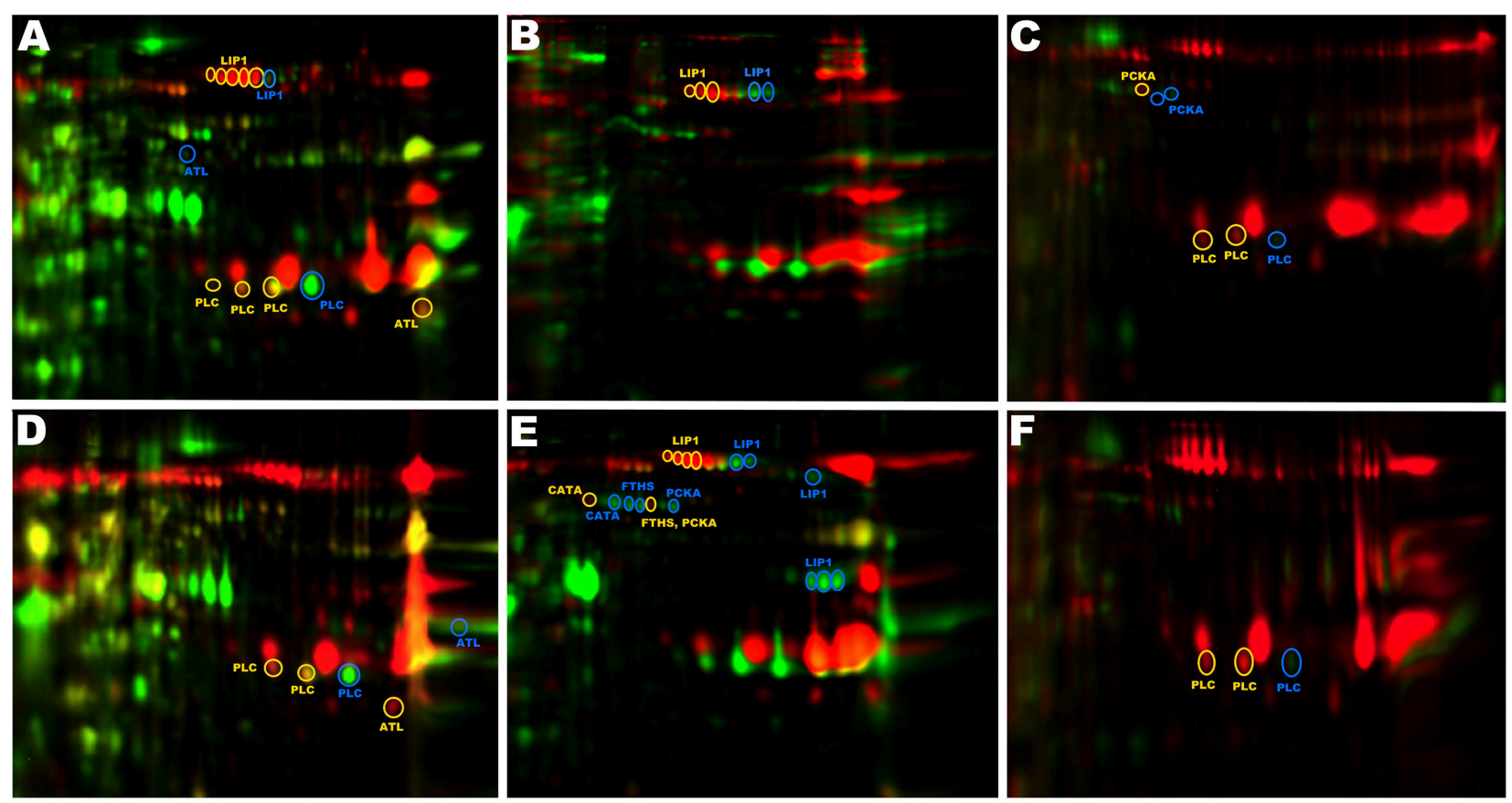

FIGURE 4 | Non-overlapping spots with identical identifications. Spots originating from the same protein do not overlap on $2 \mathrm{D}$ gels due to genetic variation and differential posttranslational processing. (A) CH3/ch24, (B) CH3/pa3, (C) CH3/ph1, (D) CH5/ch24, (E) CH5/pa3, (F) CH5/ph1. Red (yellow circles) - VIR strain; green (blue circles) - NVIR strain; orange (yellow circles) - overlapping spots.

as originating from a single protein also indicates their high expression. On the other hand, the NVIR exoproteomes are more complex (contain a larger number of different proteins), indirectly suggesting that commensal colonization possibly requires finer tune up compared to brute force pathogenesis. The last conclusion is however highly speculative.

The analyzed exoproteomes were very heterogeneous. For example, the exoproteome of the virulent strain $\mathrm{CH} 23$ differed only in five proteins from the exoproteome of the non-virulent strain ch22, whereas in another pair $(\mathrm{CH} 5 / \mathrm{ch} 24) 42$ differentiating proteins were identified. Significant heterogeneity within the exoproteomes was, however, expected, given the high plasticity of staphylococcal genomes, and was experimentally documented previously (Ziebandt et al., 2010), while we were interested in identifying virulence patterns/markers within this heterogeneity. Comparison of the VIR and NVIR proteomes identified HLA as a possible marker of the former group. HLA (haemolysin alpha; alpha toxin) is a pore forming toxin penetrating host cell membranes and resulting in their lysis. Studies in animal models of staphylococcal keratitis (McCormick et al., 2009) and pneumonia (Bubeck Wardenburg et al., 2007) document an important role of HLA in virulence, nevertheless its role in avian infection models has not been directly tested. It is known, however, that HLA uses ADAM 10 as a receptor and HLA-driven toxicity depends on the amount of this receptor which differs among cell types and/or the source species (Berube and Bubeck Wardenburg, 2013). High expression of ADAM 10 documented in many tissues of the chicken embryo (Hall and Erickson, 2003), including epidermis during formation of feather buds (Lin et al., 2011) indirectly suggests possible involvement of HLA in staphylococcal virulence in this specie, however direct evidence is missing. Secretion of HLA in in vitro cultures characterizes particular clonal types of $S$. aureus, among other, lineage CC5 (Monecke et al., 2014). This is consistent with our findings since the virulent strains tested in our study belonged to lineage CC5 (Polakowska et al., 2012). Due to the limited number of data, the production of HLA could not have been correlated with the host species (Monecke et al., 2014). However, the secretion of HLA only by the poultry-derived strains exhibiting high virulence in the chicken embryo model, as evidenced in our study, may suggest the toxin as a host-associated virulence factor; nevertheless, this is purely speculative and requires further testing.

The N-terminal domain of the major staphylococcal autolysin (ATL) was the second identified protein characteristic for all VIR strains, but not found in the NVIR exoproteomes. Interestingly, the non-virulent strains contained either the C-terminal domain of ATL (ch24 and ph2) or no ATL at all. Only in the NVIR pa3 exoproteome, the N-terminal domain and the middle part of ATL have been identified. ATL is a cell-wall associated bifunctional enzyme involved in daughter cell separation (Sugai et al., 1995; Takahashi et al., 2002). Neither the direct toxicity of ATL toward chicken cells nor the potential indirect involvement of ATL in virulence of $S$. aureus has not been investigated, according to our knowledge. It was demonstrated, however, that in closely related species, S. epidermidis and S. lugdunensis, atl-null strains are attenuated in an intravascular catheter-associated infection rat model (Rupp et al., 2001) and in a Caenorhabditis elegans model, 
respectively (Gibert et al., 2014). It is unclear, however, whether the decreased virulence was associated with an overall decreased fitness or reflected some specific function of the autolysin. ATL was also implicated in biofilm formation (Bose et al., 2012), which has an established role in staphylococcal virulence. Interestingly, we found that SSPA was expressed exclusively by the NVIR strains, while this protease has been reported to degrade ATL thus exerting negative effect on ATL mediated biofilm formation in S. aureus (Chen et al., 2013). Our data suggests SSPA mediated degradation of the N-terminal part of ATL only since the $\mathrm{N}$ terminal part of ATL was found exclusively in VIR proteomes where SSPA was not present and in the pa3 exoproteome, where SSPA was identified only in the form of an inactive zymogen.

The initial translation product of the atl gene undergoes proteolytic processing that yields two catalytically active proteins: an amidase (AM) and a glucosaminidase (GL), which corroborates the findings of this study. However, limited data is available to distinguish the roles of these two enzymatic activities in staphylococcal physiology. It has only been demonstrated that, in parallel to the enzymatic activity, AM exhibits adhesion-like vitronectin-binding activity (Heilmann et al., 1997), while GL has a DNA-binding capacity (Grilo et al., 2014). The limited amount of information does not allow us to speculate on the potential role of differential stability of AM and GL in the VIR and NVIR strains documented in this study. Nevertheless, it is worth noting that not only the expression of ATL-derived amidase was characteristic for the VIR strains. Certain VIR strains (CH3 and $\mathrm{CH} 5)$ were also characterized by expression of Y2979 protein, which contains an $\mathrm{N}$-acetylmuramoyl-L-alanine amidase domain similar to AM. Moreover, Y2979 secretion is affected by mutations in accessory secretory systems responsible for the export of staphylococcal virulence factors (Siboo et al., 2008; Sibbald et al., 2010). However, the significance of this fact remains unknown.

Besides the above-described functions, ATL was implicated in excretion of cytoplasmic proteins. A study by Pasztor et al. has shown that mutation within the atl gene affected excretion of 22 typically cytoplasmic proteins. Interestingly, the ATL related excretion was discriminatory since the most abundant cytoplasmic proteins were not found outside the cell (Pasztor et al., 2010). In our study, more cytoplasmic proteins were identified in the non-virulent exoproteome (48), compared to that of the virulent strains (28). The correlation of this fact and differential ATL stability in the VIR and NVIR strains is unknown. However, since excretion of cytosolic proteins is clearly connected with bacterial virulence (Gotz et al., 2015), the imbalance in such proteins in the exoproteomes of the VIR and NVIR strains opens a field for further research. In summary, despite data linking the presence or activity of ATL with staphylococcal phenotype its role in virulence is questionable.

Recent findings indicate that the virulence level is related not only to the expression of virulence factors but also antivirulence genes, which are characteristic for nonpathogenic strains of otherwise pathogenic bacteria (Merhej et al., 2013). While analyzing the protein spots characteristic for NVIR proteomes only, we identified SSPA expression as a fingerprint of the NVIR exoproteome. This fact was at first unexpected, since staphylococcal proteases have been widely implicated in virulence. In particular, a number of reports consistently suggested the role of SSPA in staphylococcal virulence based on certain in vitro properties and indirect observations (Prokešová et al., 1988, 1992; Karlsson et al., 2001) However, the data from in vivo models is relatively inconclusive (Coulter et al., 1998; Rice et al., 2001), clearly indicating only that although a single protease gene knock-out may not change the virulence, the orchestrated action of multiple secreted staphylococcal proteases may have an profound effect on the growth and survival of S. aureus in the infected host (Kolar et al., 2013). Therefore, advanced combination knock-outs are needed to unambiguously indicate the role of particular proteases in staphylococcal virulence. Moreover, all the above reports considered virulence in human/mammalian models, while Lowder and colleagues (Lowder et al., 2009) observed sspA pseudogenization in some poultry isolates, which indirectly suggests dispensability of SSPA in the avian host. Although the avian strains characterized in our study all contained the intact sspA gene, our data indirectly suggest the dispensability of SSPA in staphylococcal virulence in an avian host since its expression was not identified in any of the VIR strains analyzed.

SSPA is coexpressed with SSPB within a single operon (Rzychon et al., 2003). Consistently, SSPB expression was found exclusively in the NVIR strains (ch24, pa3, and ph2). Previous studies identified SSPB as an important staphylococcal virulence factor (Potempa and Pike, 2009; Smagur et al., 2009; Ohbayashi et al., 2011), but as for SSPA, the studies involved mammalian models only. Our data indicates that SSPB is dispensable for $S$. aureus virulence in the avian host since its expression was not identified in any of the virulent strains.

Besides the differential expression of multiple proteins, genetic variability (allelic variants) further contributes to the heterogeneity of staphylococcal proteomes as exemplified here by LIP1, PLC, and FTHS. This strengthens the importance of combining genomic and proteomic approaches to exoproteome analysis. The extent and impact of allelic variability on the virulence potential of staphylococci remains almost completely unexplored and is certainly worth further insight.

Post-translational modifications provide yet another source of variability within the exoproteomes. Secreted proteases are important agents modulating the extracellular as well as cellwall-bound proteome, which has important implications in staphylococcal virulence (Kantyka et al., 2013; Kolar et al., 2013). This study corroborates the previous findings on zymogen maturation, but at the same time provides evidence that proteolytic processing is more extended and complex than simple zymogen activation. This is exemplified by the differential stability of the ATL domains analyzed here in some detail, but also by the fact that the extent of zymogen processing is dissimilar in different strains, certain proteins are processed at sites other than the classical activation site, and proteins such as CATA and PCKA are processed to smaller stable fragments than what is classically considered as their mature form. Besides zymogen activation, the impact on those processes on staphylococcal physiology remains to be uncovered.

Yet another level of variability within the exoproteomes is provided by posttranslational modifications other than proteolysis. These are manifested as populations of spots 
originating from a single protein and having identical molecular weight but differing in pI. These modifications were observed in this and many other studies, but were not analyzed further and their nature remains unknown, though certainly worth further investigation.

Summarizing, despite the fact that the extracellular proteomes of the VIR and NVIR strains are heterogeneous within each group, we identified fingerprint proteins characteristic for each phenotype. Haemolysin alpha (HLA) is expressed exclusively in virulent strains and hence may be considered as a poultry-hostassociated virulence factor. Serine protease SSPA is characteristic for the exoproteomes of non-virulent strains only. It was demonstrated here that staphopain C, a protease previously implicated in staphylococcal virulence in the avian host, is alone not capable of increasing the pathogenicity of non-virulent avian strains and is dispensable in the virulent phenotype. Further, this study demonstrates that besides expression levels of different proteins, posttranslational modifications and allelic variability significantly contribute to the changeability within staphylococcal extracellular proteomes. The latter processes are largely unexplored but clearly merit in-depth characterization.

\section{AUTHOR CONTRIBUTIONS}

$\mathrm{EB}$ and BW designed the study. EB, IW, UJ, SK, MB, KP, $\mathrm{ML}, \mathrm{MK}$, and AS performed the experiments. EB, SK, KP, $\mathrm{GD}, \mathrm{AS}, \mathrm{AF}, \mathrm{JM}, \mathrm{AD}$, and $\mathrm{BW}$ analyzed and interpreted data.

\section{REFERENCES}

Alban, A., David, S. O., Bjorkesten, L., Andersson, C., Sloge, E., Lewis, S., et al. (2003). A novel experimental design for comparative two-dimensional gel analysis: two-dimensional difference gel electrophoresis incorporating a pooled internal standard. Proteomics 3, 36-44. doi: 10.1002/pmic.200 390006

Barbier, F., Lebeaux, D., Hernandez, D., Delannoy, A. S., Caro, V., Francois, P., et al. (2010). High prevalence of the arginine catabolic mobile element in carriage isolates of methicillin-resistant Staphylococcus epidermidis. J. Antimicrob. Chemother. 66, 29-36. doi: 10.1093/jac/dkq410

Becker, R. E., and Bubeck Wardenburg, J. (2015). Staphylococcus aureus and the skin: a longstanding and complex interaction. Skinmed 13, 111-119. Avaliable online at: http://www.skinmedjournal.com/

Berube, B. J., and Bubeck Wardenburg, J. (2013). Staphylococcus aureus $\alpha-$ Toxin: nearly a century of intrigue. Toxins (Basel) 5, 1140-1166. doi: 10.3390/toxins5061140

Bohach, G. A., Fast, D. J., Nelson, R. D., and Schlievert, P. M. (1990). Staphylococcal and streptococcal pyrogenic toxins involved in toxic shock syndrome and related illnesses. Crit. Rev. Microbiol. 17, 251-272. doi: 10.3109/10408419009105728

Bose, J. L., Lehman, M. K., Fey, P. D., and Bayles, K. W. (2012). Contribution of the Staphylococcus aureus Atl AM and GL murein hydrolase activities in cell division, autolysis, and biofilm formation. PLoS ONE 7:e42244. doi: 10.1371/journal.pone.0042244

Bubeck Wardenburg, J., Bae, T., Otto, M., Deleo, F. R., and Schneewind, O. (2007). Poring over pores: alpha-hemolysin and Panton-Valentine leukocidin in Staphylococcus aureus pneumonia. Nat. Med. 13, 1405-1406. doi: 10.1038/nm1207-1405

Bukowski, M., Lyzen, R., Helbin, W. M., Bonar, E., Szalewska-Palasz, A., Wegrzyn, G., et al. (2013). A regulatory role for Staphylococcus aureus toxin-antitoxin system PemIKSa. Nat. Commun. 4, 2012. doi: 10.1038/ncomms3012
EB and BW wrote the manuscript. All authors revised the manuscript and agreed to be accountable for all aspect of the work herein.

\section{FUNDING}

This research was supported by funds granted by the National Science Centre (NCN, Poland) on the basis of the decision no. DEC-2012/07/D/NZ2/04282 (to BW). Proteomics were carried out with the equipment purchased through European Union structural funds, grant POIG.02.01.00-12-167/08 (Malopolska Centre of Biotechnology).

\section{ACKNOWLEDGMENTS}

The authors thank R. Fitzgerald, A. Wieliczko, and J. Krol, for providing Staphylococcus isolates. Faculty of Biochemistry, Biophysics and Biotechnology of Jagiellonian University is a partner of the Leading National Research Center (KNOW) supported by the Ministry of Science and Higher Education, Warsaw, Poland.

\section{SUPPLEMENTARY MATERIAL}

The Supplementary Material for this article can be found online at: http://journal.frontiersin.org/article/10.3389/fcimb. 2016.00051
Chambers, H. F., and Deleo, F. R. (2009). Waves of resistance: Staphylococcus aureus in the antibiotic era. Nat. Rev. Microbiol. 7, 629-641. doi: $10.1038 /$ nrmicro2200

Chen, C., Krishnan, V., Macon, K., Manne, K., Narayana, S. V., and Schneewind, O. (2013). Secreted proteases control autolysin-mediated biofilm growth of Staphylococcus aureus. J. Biol. Chem. 288, 29440-29452. doi: 10.1074/jbc.M113.502039

Chen, Y. E., and Tsao, H. (2013). The skin microbiome: current perspectives and future challenges. J. Am. Acad. Dermatol. 69, 143-155. doi: 10.1016/j.jaad.2013.01.016

Coulter, S. N., Schwan, W. R., Ng, E. Y., Langhorne, M. H., Ritchie, H. D., Westbrock-Wadman, S., et al. (1998). Staphylococcus aureus genetic loci impacting growth and survival in multiple infection environments. Mol. Microbiol. 30, 393-404. doi: 10.1046/j.1365-2958.1998.01075.x

Devriese, L. A. (1990). Staphylococci in healthy and diseased animals. Soc. Appl. Bacteriol. Symp. Ser. 19, 71S-80S. doi: 10.1111/j.1365-2672.1990.tb01799.x

Diep, B. A., Gill, S. R., Chang, R. F., Phan, T. H., Chen, J. H., Davidson, M. G., et al. (2006). Complete genome sequence of USA300, an epidemic clone of community-acquired meticillin-resistant Staphylococcus aureus. Lancet 367, 731-739. doi: 10.1016/S0140-6736(06)68231-7

Diep, B. A., Stone, G. G., Basuino, L., Graber, C. J., Miller, A., Des Etages, S. A., et al. (2008). The arginine catabolic mobile element and staphylococcal chromosomal cassette mec linkage: convergence of virulence and resistance in the USA300 clone of methicillin-resistant Staphylococcus aureus. J. Infect. Dis. 197, 1523-1530. doi: 10.1086/587907

Duthie, E. S., and Lorenz, L. L. (1952). Staphylococcal coagulase, mode of action and antigenicity. J. Gen. Microbiol. 6, 95-107. doi: 10.1099/00221287-6-1-2-95

Gibert, L., Didi, J., Marlinghaus, L., Lesouhaitier, O., Legris, S., Szabados, F., et al. (2014). The major autolysin of Staphylococcus lugdunensis, AtlL, is involved in cell separation, stress-induced autolysis and contributes to bacterial pathogenesis. FEMS Microbiol. Lett. 352, 78-86. doi: 10.1111/1574-6968. 12374 
Gotz, F., Heilmann, C., and Stehle, T. (2014). Functional and structural analysis of the major amidase (Atl) in Staphylococcus. Int. J. Med. Microbiol. 304, 156-163. doi: 10.1016/j.ijmm.2013.11.006

Gotz, F., Yu, W., Dube, L., Prax, M., and Ebner, P. (2015). Excretion of cytosolic proteins (ECP) in bacteria. Int. J. Med. Microbiol. 305, 230-237. doi: 10.1016/j.ijmm.2014.12.021

Grilo, I. R., Ludovice, A. M., Tomasz, A., De Lencastre, H., and Sobral, R. G. (2014). The glucosaminidase domain of Atl - the major Staphylococcus aureus autolysin - has DNA-binding activity. Microbiologyopen 3, 247-256. doi: $10.1002 / \mathrm{mbo3} .165$

Hall, R. J., and Erickson, C. A. (2003). ADAM 10: an active metalloprotease expressed during avian epithelial morphogenesis. Dev. Biol. 256, 146-159. doi: 10.1016/S0012-1606(02)00133-1

Hata, E., Katsuda, K., Kobayashi, H., Uchida, I., Tanaka, K., and Eguchi, M. (2010). Genetic variation among Staphylococcus aureus strains from bovine milk and their relevance to methicillin-resistant isolates from humans. J. Clin. Microbiol. 48, 2130-2139. doi: 10.1128/JCM.01940-09

Heilmann, C., Hussain, M., Peters, G., and Gotz, F. (1997). Evidence for autolysin-mediated primary attachment of Staphylococcus epidermidis to a polystyrene surface. Mol. Microbiol. 24, 1013-1024. doi: 10.1046/j.13652958.1997.4101774.x

Kantyka, T., Pyrc, K., Gruca, M., Smagur, J., Plaza, K., Guzik, K., et al. (2013). Staphylococcus aureus proteases degrade lung surfactant protein A potentially impairing innate immunity of the lung. J. Innate Immun. 5, 251-260. doi: $10.1159 / 000345417$

Karlsson, A., Saravia-Otten, P., Tegmark, K., Morfeldt, E., and Arvidson, S. (2001). Decreased amounts of cell wall-associated protein A and fibronectinbinding proteins in Staphylococcus aureus sarA mutants due to upregulation of extracellular proteases. Infect. Immun. 69, 4742-4748. doi: 10.1128/IAI.69.8.4742-4748.2001

Kolar, S. L., Ibarra, J. A., Rivera, F. E., Mootz, J. M., Davenport, J. E., Stevens, S. M., et al. (2013). Extracellular proteases are key mediators of Staphylococcus aureus virulence via the global modulation of virulence-determinant stability. Microbiologyopen 2, 18-34. doi: 10.1002/mbo3.55

Kreiswirth, B. N., Lofdahl, S., Betley, M. J., O’reilly, M., Schlievert, P. M., Bergdoll, M. S., et al. (1983). The toxic shock syndrome exotoxin structural gene is not detectably transmitted by a prophage. Nature 305, 709-712. doi: $10.1038 / 305709 \mathrm{a} 0$

Kuramasu, S., Imamura, Y., Takizawa, T., Oguchi, F., and Tajima, Y. (1967). Studies on staphylococcosis in chickens. I. Outbreaks of staphylococcal infection on poultry farms and characteristics of Staphylococcus aureus isolated from chickens. Zentralbl Veterinarmed. B 14, 646-656. doi: 10.1111/j.14390450.1967.tb00267.x

Laemmli, U. K. (1970). Cleavage of structural proteins during the assembly of the head of bacteriophage T4. Nature 227, 680-685. doi: 10.1038/227680a0

Lin, J., Luo, J., and Redies, C. (2011). Differential regional expression of multiple ADAMs during feather bud formation. Dev. Dyn. 240, 2142-2152. doi: $10.1002 /$ dvdy. 22703

Lindsay, J. A. (2014). Staphylococcus aureus genomics and the impact of horizontal gene transfer. Int. J. Med. Microbiol. 304, 103-109. doi: 10.1016/j.ijmm.2013.11.010

Lowder, B. V., Guinane, C. M., Ben Zakour, N. L., Weinert, L. A., ConwayMorris, A., Cartwright, R. A., et al. (2009). Recent human-to-poultry host jump, adaptation, and pandemic spread of Staphylococcus aureus. Proc. Natl. Acad. Sci. U.S.A. 106, 19545-19550. doi: 10.1073/pnas.090 9285106

Lowy, F. D. (1998). Staphylococcus aureus infections. N. Engl. J. Med. 339, 520-532. doi: 10.1056/NEJM199808203390806

Malachowa, N., and DeLeo, F. R. (2010). Mobile genetic elements of Staphylococcus aureus. Cell. Mol. Life Sci. 67, 3057-3071. doi: 10.1007/s00018-010-0389-4

McCarthy, A. J., Loeffler, A., Witney, A. A., Gould, K. A., Lloyd, D. H., and Lindsay, J. A. (2014). Extensive horizontal gene transfer during Staphylococcus aureus co-colonization in vivo. Genome Biol. Evol. 6, 2697-2708. doi: 10.1093/gbe/evu214

McCormick, C. C., Caballero, A. R., Balzli, C. L., Tang, A., and O'callaghan, R. J. (2009). Chemical inhibition of alpha-toxin, a key corneal virulence factor of Staphylococcus aureus. Invest. Ophthalmol. Vis. Sci. 50, 2848-2854. doi: 10.1167/iovs.08-3157
Melehani, J. H., James, D. B., Dumont, A. L., Torres, V. J., and Duncan, J. A. (2015). Staphylococcus aureus Leukocidin A/B (LukAB) kills human monocytes via host NLRP3 and ASC when extracellular, but not intracellular. PLoS Pathog. 11:e1004970. doi: 10.1371/journal.ppat.1004970

Merhej, V., Georgiades, K., and Raoult, D. (2013). Postgenomic analysis of bacterial pathogens repertoire reveals genome reduction rather than virulence factors. Brief. Funct. Genomics 12, 291-304. doi: 10.1093/bfgp/elt015

Minden, J. S., Dowd, S. R., Meyer, H. E., and Stuhler, K. (2009). Difference gel electrophoresis. Electrophoresis 30(Suppl. 1), S156-S161. doi: 10.1002/elps.200900098

Monecke, S., Muller, E., Buchler, J., Stieber, B., and Ehricht, R. (2014). Staphylococcus aureus in vitro secretion of alpha toxin (hla) correlates with the affiliation to clonal complexes. PLoS ONE 9:e100427. doi: 10.1371/journal.pone.0100427

Moore, P. C., and Lindsay, J. A. (2001). Genetic variation among hospital isolates of methicillin-sensitive Staphylococcus aureus: evidence for horizontal transfer of virulence genes. J. Clin. Microbiol. 39, 2760-2767. doi: 10.1128/JCM.39.8.27602767.2001

Nickerson, N. N., Prasad, L., Jacob, L., Delbaere, L. T., and McGavin, M. J. (2007). Activation of the SspA serine protease zymogen of Staphylococcus aureus proceeds through unique variations of a trypsinogen-like mechanism and is dependent on both autocatalytic and metalloprotease-specific processing. $J$. Biol. Chem. 282, 34129-34138. doi: 10.1074/jbc.M705672200

Ohbayashi, T., Irie, A., Murakami, Y., Nowak, M., Potempa, J., Nishimura, Y., et al. (2011). Degradation of fibrinogen and collagen by staphopains, cysteine proteases released from Staphylococcus aureus. Microbiology 157, 786-792. doi: 10.1099/mic.0.044503-0

Pasztor, L., Ziebandt, A. K., Nega, M., Schlag, M., Haase, S., Franz-Wachtel, M., et al. (2010). Staphylococcal major autolysin (Atl) is involved in excretion of cytoplasmic proteins. J. Biol. Chem. 285, 36794-36803. doi: 10.1074/jbc.M110.167312

Polakowska, K., Lis, M. W., Helbin, W. M., Dubin, G., Dubin, A., Niedziolka, J. W., et al. (2012). The virulence of Staphylococcus aureus correlates with strain genotype in a chicken embryo model but not a nematode model. Microbes Infect. 14, 1352-1362. doi: 10.1016/j.micinf.2012.09.006

Potempa, J., and Pike, R. N. (2009). Corruption of innate immunity by bacterial proteases. J. Innate Immun. 1, 70-87. doi: 10.1159/000181144

Prokešová, L., Potužníková, B., Potempa, J., Zikán, J., Radl, J., Hachová, L., et al. (1992). Cleavage of human immunoglobulins by serine proteinase from Staphylococcus aureus. Immun. Lett. 31, 259-266. doi: 10.1016/01652478(92)90124-7

Prokešová, L., Porwit-Bóbr, Z., Baran, K., Potempa, J., and John, C. (1988). Effect of serine proteinase from Staphylococcus aureus on in vitro stimulation of human lymphocytes. Immun. Lett. 19, 127-132. doi: 10.1016/0165-2478(88)90131-9

Rice, K., Peralta, R., Bast, D., De Azavedo, J., and McGavin, M. J. (2001). Description of staphylococcus serine protease (ssp) operon in Staphylococcus aureus and nonpolar inactivation of sspA-encoded serine protease. Infect. Immun. 69, 159-169. doi: 10.1128/IAI.69.1.159-169.2001

Rupp, M. E., Fey, P. D., Heilmann, C., and Gotz, F. (2001). Characterization of the importance of Staphylococcus epidermidis autolysin and polysaccharide intercellular adhesin in the pathogenesis of intravascular catheter-associated infection in a rat model. J. Infect. Dis. 183, 1038-1042. doi: 10.1086/ 319279

Rzychon, M., Sabat, A., Kosowska, K., Potempa, J., and Dubin, A. (2003). Staphostatins: an expanding new group of proteinase inhibitors with a unique specificity for the regulation of staphopains, Staphylococcus spp. cysteine proteinases. Mol. Microbiol. 49, 1051-1066. doi: 10.1046/j.13652958.2003.03613.x

Shevchenko, A., Wilm, M., Vorm, O., and Mann, M. (1996). Mass spectrometric sequencing of proteins silver-stained polyacrylamide gels. Anal. Chem. 68, 850-858. doi: $10.1021 /$ ac950914h

Shukla, S. K., Rose, W., and Schrodi, S. J. (2015). Complex host genetic susceptibility to Staphylococcus aureus infections. Trends Microbiol. 23, 529-536. doi: 10.1016/j.tim.2015.05.008

Sibbald, M. J., Winter, T., Van Der Kooi-Pol, M. M., Buist, G., Tsompanidou, E., Bosma, T., et al. (2010). Synthetic effects of secG and secY2 mutations on exoproteome biogenesis in Staphylococcus aureus. J. Bacteriol. 192, 3788-3800. doi: $10.1128 / J B .01452-09$ 
Siboo, I. R., Chaffin, D. O., Rubens, C. E., and Sullam, P. M. (2008). Characterization of the accessory Sec system of Staphylococcus aureus. J. Bacteriol. 190, 6188-6196. doi: 10.1128/JB. 00300-08

Smagur, J., Guzik, K., Bzowska, M., Kuzak, M., Zarebski, M., Kantyka, T., et al. (2009). Staphylococcal cysteine protease staphopain B (SspB) induces rapid engulfment of human neutrophils and monocytes by macrophages. Biol. Chem. 390, 361-371. doi: 10.1515/BC.2009.042

Smyth, D. S., Feil, E. J., Meaney, W. J., Hartigan, P. J., Tollersrud, T., Fitzgerald, J. R., et al. (2009). Molecular genetic typing reveals further insights into the diversity of animal-associated Staphylococcus aureus. J. Med. Microbiol. 58, 1343-1353. doi: 10.1099/jmm.0.009837-0

Spaan, A. N., Schiepers, A., De Haas, C. J., Van Hooijdonk, D. D., Badiou, C., Contamin, H., et al. (2015). Differential interaction of the Staphylococcal toxins panton-valentine leukocidin and gamma-hemolysin CB with human C5a receptors. J. Immunol. 195, 1034-1043. doi: 10.4049/jimmunol. 1500604

Sugai, M., Komatsuzawa, H., Akiyama, T., Hong, Y. M., Oshida, T., Miyake, Y., et al. (1995). Identification of endo-beta-Nacetylglucosaminidase and $\mathrm{N}$-acetylmuramyl-L-alanine amidase as cluster-dispersing enzymes in Staphylococcus aureus. J. Bacteriol. 177, 1491-1496.

Sung, J. M., Lloyd, D. H., and Lindsay, J. A. (2008). Staphylococcus aureus host specificity: comparative genomics of human versus animal isolates by multi-strain microarray. Microbiology 154, 1949-1959. doi: 10.1099/mic.0.2007/015289-0

Takahashi, J., Komatsuzawa, H., Yamada, S., Nishida, T., Labischinski, H., Fujiwara, T., et al. (2002). Molecular characterization of an atl null mutant of Staphylococcus aureus. Microbiol. Immunol. 46, 601-612. doi: 10.1111/j.13480421.2002.tb02741.x

Takeuchi, S., Kinoshita, T., Kaidoh, T., and Hashizume, N. (1999). Purification and characterization of protease produced by Staphylococcus aureus isolated from a diseased chicken. Vet. Microbiol. 67, 195-202. doi: 10.1016/S03781135(99)00034-6
Takeuchi, S., Matsunaga, K., Inubushi, S., Higuchi, H., Imaizumi, K., and Kaidoh, T. (2002). Structural gene and strain specificity of a novel cysteine protease produced by Staphylococcus aureus isolated from a diseased chicken. Vet. Microbiol. 89, 201-210. doi: 10.1016/S0378-1135(02)00171-2

Timms, J. F., and Cramer, R. (2008). Difference gel electrophoresis. Proteomics 8 , 4886-4897. doi: 10.1002/pmic.200800298

Traber, K. E., Lee, E., Benson, S., Corrigan, R., Cantera, M., Shopsin, B., et al. (2008). agr function in clinical Staphylococcus aureus isolates. Microbiology 154, 2265-2274. doi: 10.1099/mic.0.2007/011874-0

Traber, K., and Novick, R. (2006). A slipped-mispairing mutation in AgrA of laboratory strains and clinical isolates results in delayed activation of agr and failure to translate delta- and alpha-haemolysins. Mol. Microbiol. 59, 1519-1530. doi: 10.1111/j.1365-2958.2006.04986.x

Viana, D., Blanco, J., Tormo-Mas, M. A., Selva, L., Guinane, C. M., Baselga, R., et al. (2010). Adaptation of Staphylococcus aureus to ruminant and equine hosts involves SaPI-carried variants of von Willebrand factor-binding protein. Mol Microbiol. 77, 1583-1594. doi: 10.1111/j.1365-2958.2010.07312.x

Ziebandt, A. K., Kusch, H., Degner, M., Jaglitz, S., Sibbald, M. J., Arends, J. P. et al. (2010). Proteomics uncovers extreme heterogeneity in the Staphylococcus aureus exoproteome due to genomic plasticity and variant gene regulation. Proteomics 10, 1634-1644. doi: 10.1002/pmic.200900313

Conflict of Interest Statement: The authors declare that the research was conducted in the absence of any commercial or financial relationships that could be construed as a potential conflict of interest.

Copyright (c) 2016 Bonar, Wojcik, Jankowska, Kedracka-Krok, Bukowski Polakowska, Lis, Kosecka-Strojek, Sabat, Dubin, Friedrich, Miedzobrodzki, Dubin and Wladyka. This is an open-access article distributed under the terms of the Creative Commons Attribution License (CC BY). The use, distribution or reproduction in other forums is permitted, provided the original author(s) or licensor are credited and that the original publication in this journal is cited, in accordance with accepted academic practice. No use, distribution or reproduction is permitted which does not comply with these terms. 